\title{
Unusual Fibrosclerotic Lesion of the Laryngotracheal Complex Presenting as Subglottic Stenosis
}

\begin{abstract}
Douglas R. Johnston, MD; Joseph M. Curry, MD; Raphael Rubin, MD; Marc R. Rosen, MD

We present a case report that describes the pathology, presentation, and management complexities of an unusual, destructive fibrosclerotic lesion of the laryngotracheal complex. An otherwise healthy 21-year-old man presented with a 1-year history of progressive shortness of breath and stridor. The initial examination revealed a 3-cm, grade III subglottic stenosis. Nodular fibrosis of the strap muscles, laryngotracheal cartilages, and trachea was evident. Biopsies revealed dense peritracheal desmoplastic reaction with focal erosion of cartilage. However, features diagnostic for relapsing polychondritis, desmoid tumor, or orbital pseudotumor were absent. The disease progressed to involve severe stenosis and thickening of the trachea and main stem bronchi. Surgical and medical management of this unusual fibrosclerotic lesion did not ameliorate the disease process, but a recent encouraging response to tamoxifen citrate has been observed with improvements in vocal fold motion and activity levels. Prognosis and management experience for this unknown pathologic entity are absent in the literature. In this case, diffuse disease progression occurred despite surgical and medical management, but has been halted by tamoxifen therapy. The prospect of a durable response and disease remission is unknown.
\end{abstract}

Key Words: fibrosclerosis, relapsing polychondritis, subglottic stenosis, tumefactive fibroinflammatory lesion.

\section{INTRODUCTION}

Subglottic stenosis is a rare entity in the absence of a history of prior intubation, tracheotomy, or other airway instrumentation. Stenoses without a history of local trauma usually appear shortly after birth. Disease processes known to cause idiopathic subglottic stenosis are mainly autoimmune in nature and include relapsing polychondritis, sarcoidosis, systemic lupus erythematosus, Wegener's granulomatosis, Behçet's syridrome, and other rare conditions. The mainstay of surgical treatment for these entities is endoscopic laser treatment, dilation, and tracheotomy for severe stenosis, whereas medical therapies consist largely of systemic corticosteroids and antimetabolites. Because of the unknown cause of the disease process in the patient presented here, the established treatment and prognosis have not been defined. Continued disease progression to the entire trachea in this case has essentially elimunated surgical treatment options and focused the treating physicians' attention on medical treatment options.

\section{CASE REPORT}

We present the case of a 21-year-old man who presented with a 1-year history of progressive shortness of breath. He had been evaluated at an outside insti- tution for this respiratory difficulty and had received a diagnosis of asthma and had undergone treatment with bronchodilators. To this point in time, he was otherwise healthy and active. His history included a 2-week intubation in infancy, but he recovered uneventfully and had a healthy childhood without other airway problems. He was stridorous on presentation, and nasopharyngolaryngoscopy revealed a marginal airway and severely restricted vocal fold motion. He promptly underwent fiberoptic intubation and tracheotomy with bronchoscopy. Tissues of the larynx, trachea, thyroid, and strap muscles were noted to be involved in a dense, nodular inflammatory process. Stenosis of the laryngeal inlet was identified with an approximately $0.5-\mathrm{cm}$ transverse Cotton-Myer grade III subglottic stenosis (Fig 1). The stenotic region widened as it extended distally for approximately $3 \mathrm{~cm}$, and computed tomography revealed irregular tracheal calcification (Fig 2).

Tracheal biopsies displayed a dense peritracheal desmoplastic reaction and chronic inflammatory infiltrate with focal erosion of cartilage (Fig 3). A thyroid biopsy was characterized by a fibromyxoid proliferation.

Rheumatologic consultation was obtained to aid in medical management. Autoimmune serologic

\footnotetext{
From the Departments of Otolaryngology (Johnston, Curry, Rosen) and Pathology, Anatomy and Cell Biology (Rubin), Thomas Jefferson University Hospital, Philadelphia, Pennsylvania.

Correspondence: Marc R. Rosen, MD, Thomas Jefferson University, Dept of Otolaryngology-Head and Neck Surgery, 925 Chestnut St, 6th Floor, Philadelphia, PA 19107.
} 


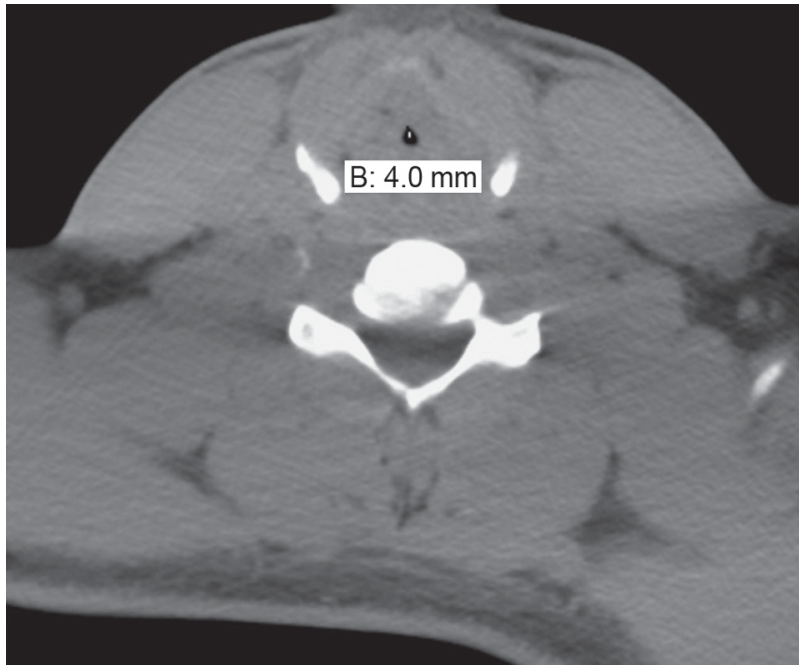

Fig 1. Subglottic narrowing shows Cotton-Myer stage III stenosis.

testing was nondiagnostic (erythrocyte sedimentation rate, antinuclear antibody, anti-single-stranded DNA antibody, anti-double-stranded DNA antibody, anti-Smith antibody, anti-SS-A antibody, anti-SS-B antibody, antineutrophil cytoplasmic antibody with cytoplasmic and perinuclear staining, rheumatoid factor, and C-reactive protein). The patient was placed on a regimen of high-dose prednisone, and he initially responded to a 3-month course with improved vocal fold mebility and improved subglottic stenosis. As the steroid dosage was tapered, however, the clinical findings stabilized and then worsened. The limited vocal fold motion was thought to be a result of bilateral disease extension to either the recurrent laryngeal nerves or the cri-

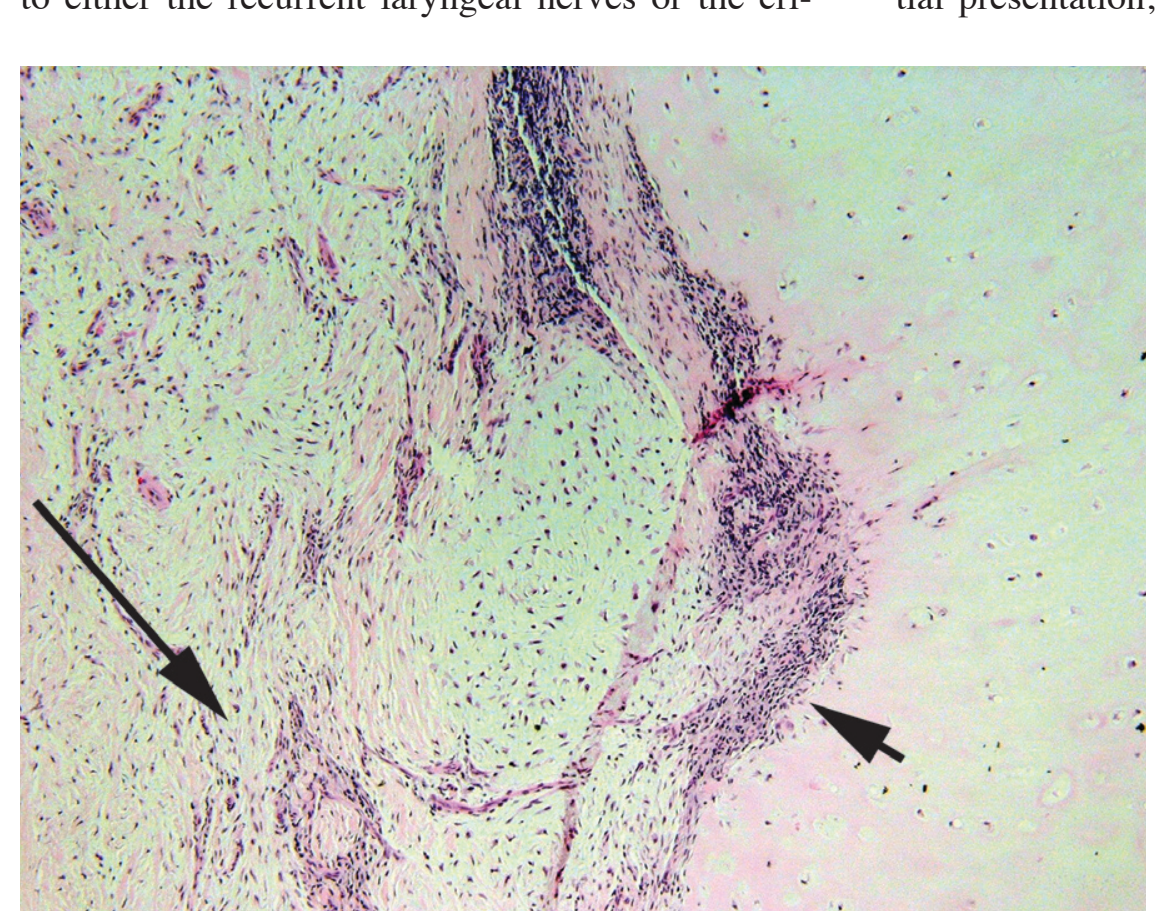

Fig 3. Tracheal biopsy shows erosion of cartilage by chronic inflammatory infiltrate (short arrow), and fibromyxoid change within adjacent soft tissue (long arrow). 
ameter above the carina was approximately $1.6 \mathrm{~cm}$ and the disease was limited to the glottis and subglottis. However, 17 months after the initial imaging, the anterior-posterior diameter above the carina had narrowed to $8 \mathrm{~mm}$.

Therapy with the antiestrogen agent tamoxifen citrate was started approximately 8 months ago, with rapid improvement in shortness of breath and slightly improved vocal fold mobility. However, acute worsening in shortness of breath 6 months ago prompted operative flexible bronchoscopy that revealed granulation tissue at the site of the tracheotomy tube tip, which was relieved with flexible laser treatment. Bronchoscopy showed continued severe tracheal stenosis and worsening left main stem bronchial stenosis that would not permit the passage of the 6-mm scope. The tamoxifen therapy was continued despite this relapse of symptoms, and referral to a pulmonologist was made. Although the response to the tamoxifen has not been consistent, the patient remains on tamoxifen and, 8 months after initiation of therapy, enjoys improved vocal fold motion and greatly improved activity levels, such that he is once again participating in recreational athletics. Curiously, recent bronchoscopy has not demonstrated improvement in the tracheal or main stem bronchial stenosis.

\section{DISCUSSION}

This unusual lesion is pathologically distinct from previously described non-neoplastic diseases of the laryngotracheal complex. Relapsing polychondritis presents with tracheomalacia and inflammation with pleomorphic lymphocytic infiltrate, which were not characteristic of this lesion. Additionally, the lesion did not exhibit the more cellular appearance of desmoid tumor. ${ }^{2}$ The prominent mononuclear infiltrate of inflammatory pseudotumor and tumefactive fibroinflammatory lesions of the head and neck was also absent. ${ }^{3-7}$ An outside review of the pathologic findings confirmed the unusual features of this lesion, but did not establish a firm diagnosis.

Because this disease process is unrecognized, the literature does not contain prognostic or therapeutic experience. Initial hopes of decannulation after periods of disease response were not realized, because of poor vocal fold motion and worsening stenosis. Because the trachea has become diffusely involved, including main stem bronchial thickening and narrowing, simple excision of the stenotic segment is not an option. Serial lasering and dilations with the addition of a topical agent to prevent rescarring, such as the antimetabolite mitomycin $\mathrm{C}$, are also not feasible alternatives, because of the distal extent of disease. The disease progression that occurred through surgical management and trials of systemic corticosteroids and penicillamine portended poorly for future decannulation and normal activity levels. The antiestrogen drug tanoxifen, however, has halted disease progression at this point and has afforded mildly improved yocal fold function and greatly improved activity levels. The durability of this improvement remains to be seen, especially in light of the persistent tracheal and main stem bronchial narrowing.

The response to tamoxifen may be secondary to its antifibroblast activity. ${ }^{8}$ Additionally, estrogen receptors have been shown to be present in the human trachea.9 Other fibroproliferative disease processes have responded favorably to tamoxifen therapy, such as retroperitoneal fibrosis. ${ }^{10}$ Tamoxifen therapy will continue, along with serial visits and examinations, but further surgical management is not currently warranted. The patient may be a candidate for transitioning to a T-tube for ease in care and to stent the subglottis, but given the still-limited vocal fold motion, decannulation is not an option.
1. Jones KR. Infections and systemic manifestations of disease of the larynx. In: Cummings CW, Flint PW, Haughey BH, et al, eds. Cummings' otolaryngology-head and neck surgery. 4th ed. Philadelphia, Pa: Mosby, 2005:2065-76.

2. Wang CP, Chang YL, Ko JY, Cheng CH, Yeh CF, Lou PJ. Desmoid tumor of the head and neck. Head Neck 2006;28:100813.

3. Wold LE, Weiland LH. Tumefactive fibro-inflammatory lesions of the head and neck. Am J Surg Pathol 1983;7:477-82.

4. Olsen KD, DeSanto LW, Wold LE, Weiland LH. Tumefactive fibroinflammatory lesions of the head and neck. Laryngoscope 1986;96:940-4.

5. Weisberger EC, Zauel DW, Gilmor RL, Yune H. Otolaryngologists' role in diagnosis and management of orbital pseudotumor. Otolaryngol Head Neck Surg 1985;93:536-49.
6. Dunn EJ, Ulicny KS Jr, Wright CB, Gottesman L. Surgical implications of sclerosing mediastinitis. A report of six cases and review of the literature. Chest 1990;97:338-46.

7. Sekine S, Nagata M, Watanabe T. Chronic sclerosing sialadenitis of the submandibular gland associated with idiopathic retroperitoneal fibrosis. Pathol Int 1999;49:663-7.

8. Kuhn MA, Wang X, Payne WG, Ko F, Robson MC. Tamoxifen decreases fibroblast function and downregulates TGF(beta2) in Dupuytren's affected palmar fascia. J Surg Res 2002;103:146-52.

9. Ben-Hur H, Thole HH, Mashiah A, et al. Estrogen, progesterone and testosterone receptors in human fetal cartilaginous tissue: immunohistochemical studies. Calcif Tissue Int 1997;60:520-6.

10. Vaglio A, Salvarani C, Buzio C. Retroperitoneal fibrosis. Lancet 2006;367:241-51. 